\title{
Study on Standardization of Term Translation in Petroleum Industry--- Take the Term Translation in Technical Supervision in Petroleum Industry as Example
}

\author{
Huan-Ting SUN ${ }^{a}$, Ke CHEN ${ }^{b}$ \\ Xi'an Shiyou University, Xi'an, Shaanxi Province, 710065, P.R China \\ a2518696341@qq.com, blove200508@qq.com
}

Keywords: Standardization, Petroleum industry, Term translation.

\begin{abstract}
Terms reflect the characteristic of all trades and professions, therefore the standardization of term translation is not only the need of industries to publicize themselves, but also the basis for their intercommunication. This paper selected certain terms in the Technical Supervision of Petroleum Industry published in 2015. Based on the analysis of their translations, the paper proposes translations preferred with consideration of the basic characteristics of a term as well as word composition.
\end{abstract}

Society developing, new concepts are emerging in different industries. This requires the concepts must be made and defined scientifically for standardized use and management when terms came into being correspondingly. What is a term? A term is a collection of appellations used to represent concepts in a particular subject, and a conventional language symbol that expresses or defines scientific concepts through speech or words, working as a tool for the exchange of ideas and thoughts. As a term can not be used arbitrarily, so it can not be translated carelessly. A terms and its translation need to be standardized. To standardize terms is to standardize the vocabulary in a particular field so that one of the terms corresponds to one concept and only one concept so as to reduce the non-standard use of a term out of polysemy. Standardized terms are based on the standardization of terms, providing better storage and specification of information, and helping coordination of concepts and terms. Accordingly, accuracy and rigor are in need for translation of a term Otherwise, the concepts will be confusing, and the allegation unclear, meanings misunderstood.

In 2015, the Technical Supervision of petroleum industry released a total of 12 issues with 768 key words, among which 650 words are the targets for improvement. There are 20 pairs of inconsistencies in translation, of which 13 are the same in Chinese but different in English, and the remaining 7 pairs are in the same English but different in Chinese terms. Now this paper is to analyze relevant terms and put forward personal reference translation, with reference to the basic characteristics of terms and consultation of the Dictionary of English-Chinese Petroleum (hereinafter referred to as the "Dictionary") and Chinese-English Petroleum Technology Dictionary (hereinafter referred to as "Technology Dictionary ").

\section{The Basic Characteristics of a Term}

A few basic characteristics of term are necessarily explained before the analysis to be done:

(1)Professional. A term is to express the special concepts of various professions, so the scope of use is limited, and the need is little.

(2)Scientific. A term has a semantic range that is accurate not only to mark one concept but also to make it precise and to distinguish it from similar concepts.

(3)Univocal. The key difference between terms and general vocabulary lies in univocal nature of a term, that is, it is unambiguous within the scope of a particular profession.

(4)Systematic. In a science or technology, the status of every single term can only be defined in the entire conceptual system of that profession.

(5)Concise: The exchange of information requires terms to be as concise as possible in order to 
increase efficiency.

\section{Some Terms in Dispute and to be Suggested}

After comparing the Chinese and English keywords of each thesis in Technical Supervision of Oil Industry (No. 12, 2015), it is found that some of these terms are somewhat open to question. Now a few are summarized in the following statement.

First of all, there are several problems that need to be indicated in the translation of key words in the edition (Table 1):

\section{Terms in the Same Chinese are Different in English; the Differences can be Categorized as Follows:}

(1) Different choices of words, such as "subsea pipeline" and "submarine pipeline”.

According to the note in Great Dictionary, the standard translation of "haidi guandao" should be "submarine pipeline" (P1363), whereas "subsea pipeline" refers to "haidi guanxian" (P1365). The scientific nature of terms requires that a term has a semantic range accurate not only to mark a concept but also to make it precise and to distinguish it from similar concepts so that the translator should carefully select the vocabulary for the article and avoid mixing of similar terms.

(2) The use of punctuation, such as "low permeabiltiy oilfield" and "low-permeability oilfield".

There are many kinds of word building in technical terms. In composition, the modifiers to a center word can be played by words of different parts of speech---adjectives, nouns, or nouns. According to the conciseness of terms and the method of word building, a hyphen between two words can be omitted, so the expression of a term will be more concise.

(3) Changes in the structure of the term composition, such as "proppant for fracturing" and "fracturing proppant".

According to the entry in Great Dictionary (P595) and Technical Dictionary (P1199), the term for "yalie zhichengji" (in Chinese) is "fracturing proppant'. Although the translation is analyzed from the perspective of syntactic structure, and the preposition "for" is used to indicate the relationship between the antecedents and the succeeding words, according to the conciseness of the terms, efficiency is to be guaranteed when the translation should be as simple and straightforward as possible by using gerund for center word.

(4) Different selections of parts of the selection, such as "hydrostatic testing" and "hydrostatic pressure test".

"testing" is a gerund while "test" is a simple noun. The former tends to describe dynamically, the latter prefers to show statically. Great Dictionary (P715) takes "hydrostatic testing” as the standard term.

\section{Terms in Different Chinese have the Same Translation in English.}

Some terms in Chinese are translated to be the same in English out of the subtle differences between the Chinese expressions, such as "gujing gongyi” (in Chinese) and "gujing jishu” (in Chinese).

"Technique" and "technology" are two words with different emphases. "Technology" indicates something more theoretical, systematic and integrated. Although it can be translated into "jishu"(in Chinese), "technique" is more likely to be directly applied to practical applications, tips and tricks, highlighting the fine workmanship, therefore "jishu"(in Chinese) is suggested to be translated into "cementing technology".

Besides, when there are some inconsistent expressions of a Chinese term, there is the problem that certain words are added or missed, such as "daxing fudingguan”(in Chinese) and "daxing fudingyouguan” (in Chinese).

The standard translation of "large floating-roof oil tank" should be "daxing fuding youguan" (in Chinese), but its English translation needs to be corrected so that the hyphen can be omitted, ie " large floating roof oil tank".

Second, some terms are not translated correctly. 
On the one hand, such terms are translated with improper selection of words, resulting in the deviation of meaning. On the other hand, some terms translated in a literal way results in other terms with different meanings. Such as "parallel conductance".

"Conductance" is can be both conductivity and conductivity, and when it is to say “conductivity”, it should be "conduction". Then "Conductance” for "daodian”(in Chinese) will lead to deviation in concept.

"gongyi liucheng" (in Chinese) parallel conductance should be "process flow", (P1128, Dictionary and P389, Technical Dictionary). "Technological process” shows more about steps. (P389, Technical Dictionary)

The term, "hanyou wushui" (in Chinese), is "oily sewage”, oily water or oil-bearing waste water (P990, Great Dictionary). "Oily waste water" refers to "hanyou feishui" (in Chinese) (P990, Great Dictionary). "Wastewater "and "sewage" should be two completely different industrial concepts, so the method or process of disposal should be different.

The standard term for "wusun jiance" (in Chinese) is "nondestructive examination" (P1132, Technical Dictionary). The corresponding term in Chinese for "nondestructive testing" is: NDT, also known as nondestructive testing (P960, Great Dictionary).

“chushaliang yuce” (in Chinese) ---“sand production prediction” in English--- is mainly to predict the amount of sand produced; its standard translation should be "sand production rate prediction" (P140, Technical Dictionary). "Sand production prediction" only tells the prediction about whether the sand is produced or not. If so, two translations should be completely different in nature.

"Flow capacity" means liquid flow capacity in drilling, also known as liquidity (P570, Great Dictionary); “daoliu” (in Chinese) ---“backwash" in English--- has not been included into the dictionary as an entry. With the consideration of the closeness in Chinese meaning, "backwash" should be similar to "backflow and counterflow". Given this, "daoliu" (in Chinese) can be translated into "backwash or counterflow".

The exact translation of "heat loss" is "resunshi" (in Chinese), also known as "rehaosun" (in Chinese) (P672, Great Dictionary). In petroleum industry, it is better not to use "loureliang” (in Chinese).

In Chinese composition, “pengzhangguan” (in Chinese)---“expansion tube” in English--- is in the form of a noun with a modifier to a center word, but the systematic nature of a term requires that the status of each term in a science or technology be defined only in the entire conceptual system of the profession, so it is best to select the standard translation provided by the dictionary, ie Bulged tube, expansible tube (P819, Technical Dictionary).

The univocal nature of a term indicates that the greatest difference between a term and a general word is its univocal character, ie, it is unambiguous within the scope of a particular profession. Although a vessel may also refer to a larger ship or so, there are certain standard terms that should be used when there are defined terms in the trade dictionaries. "Pipelayer" is translated as "Pipelaying barge" in Technical Dictionary (P740), so the term can be "pipelaying barge method".

In Technology Dictionary (P950) and Great Dictionary (P1272) "zhengdingyali” (in Chinese) is also known as "constant pressure", so here the term should be this.

According to the Great Dictionary (P1430), the standard translation of tight formations of natural gas is "tight gas", so "dense oil” is suggested to be "tight oil" as its standard term.

\section{Summary}

Nothing can be accomplished without norms or standards. A set of strict rules and regulations are foundation for the development of all walks of life; similarly, the proper use of terms must also follow the standards required by industries. Terms represent the characteristics of each industry, specifically bound in each, but creating a safe barrier for each. Standardization of terms is crucial to avoid unnecessary troubles caused by misuse of the terms or lack thereof. With standardized terms as the basis, communication of all walks of life and trade exchanges among nations can have institutional guarantees. 


\section{Acknowledgement}

This research was financially supported by the National Science Foundation.

\section{Reference}

[1] Dictionary of English-Chinese Petroleum, Petroleum Industry Press,2001 (in Chinese)

[2] Chinese-English Petroleum Technology Dictionary, the $3^{\text {rd }}$ edition, Petroleum Industry Press,2013 (in Chinese)

[3] Study on Terms and Term Translation, Lin-Hai Rong, Southeast University Press,2012 (in Chinese)

[4] Guide to Study on Term Translation, Xiang-Qing Wei, Lian-Zhen Zhao, Nanjing University Press,2012 (in Chinese)

[5] Research and Exploration on Chinese Terminology, Qing Liu, Commercial Press,2010 (in Chinese) 